MATHEMATICS OF COMPUTATION

Volume 68, Number 226, April 1999, Pages 585-606

S 0025-5718(99)01030-3

\title{
CONVERGENCE ANALYSIS OF DOMAIN DECOMPOSITION ALGORITHMS WITH FULL OVERLAPPING FOR THE ADVECTION-DIFFUSION PROBLEMS
}

\author{
P. LE TALLEC AND M. D. TIDRIRI
}

\begin{abstract}
The aim of this paper is to study the convergence properties of a time marching algorithm solving advection-diffusion problems on two domains using incompatible discretizations. The basic algorithm is first described, and theoretical and numerical results that illustrate its convergence properties are then presented.
\end{abstract}

\section{INTRODUCTION}

Domain decomposition methods have become an efficient strategy for solving large scale problems on parallel computers [3]-[8]. Nevertheless, they can also be used in order to couple different models $[1,9,10,12,13,16,18,19,20]$. In this paper we shall examine a domain decomposition strategy which can be applied to such situations.

This approach was introduced in order to resolve several difficulties that occur in fluid mechanics and kinetic theory. In particular, our aim is to introduce several subdomains in order to locally introduce an enriched model next to a domain boundary for an exterior domain problem. For this purpose, we propose to fully overlap subdomains and to couple the solutions through natural "friction" (Neumann) forces acting on the internal boundary of the domain, these friction forces being updated inside the time marching algorithm used for the solution of the initial boundary value problem. In the present study, only the case of two subdomains will be considered.

The theoretical study of our method will be done on an advection-diffusion problem, which will serve as our model problem in this paper. The analysis will be made at the continuous level, independently of any discretization strategy, which means that the results will be mesh independent.

In $[21,22]$ the authors studied overlapping methods based on an explicit Schwarz additive methods for time evolution parabolic problems. These methods solve the algebraic systems arising from the discretization of the unsteady parabolic problems via implicit schemes, by constructing preconditioners based on a Schwarz additive

\footnotetext{
Received by the editor January 11, 1995 and, in revised form, April 5, 1996 and November 21, 1996.

1991 Mathematics Subject Classification. Primary 65Jxx, 65M12, 65C20, 76Nxx, 82Cxx.

This work has been supported by the Hermes Research program under grant number RDAN $86.1 / 3$. The second author was also supported by the National Science Foundation under contract number ECS-8957475 and by the United Technologies Research Center while he was at Yale University.
} 
method. Our approach is quite different in that we define our method by imposing friction (Neumann) type boundary conditions on the internal boundary of the global domain and Dirichlet boundary conditions on the external boundary of the local domain at the continuous level (see Section 2). This permits us to uncouple the global problem and the local one. Hence, they can be discretized by two independent approximation methods.

In the next section we shall describe this model problem. In the third section we present our algorithm for some basic cases. In the fourth section we show how this algorithm can be applied to some problems in mechanics and kinetic theory. The fifth section deals with one-dimensional stationary problems. We will show also that the convergence of this method can be improved by introducing a relaxation parameter [5]. In the sixth section we study the linear convergence of the implicit version of the coupling algorithm in the general multidimensional case. In the last section we study the numerical stability of the explicit algorithm. Practical applications of the proposed algorithm to real life CFD problems can be found in [1], [18], [19], and [20].

\section{The model Problem}

Consider a bounded domain $\Omega$ of $\mathbb{R}^{n}$ such that its boundary $\partial \Omega$ is lipschitzian, and a connected domain $\Omega_{l o c}$ of $\mathbb{R}^{n}$ with $\Omega_{l o c} \subset \Omega$ (Figure 1). The boundaries of the two subdomains are defined as follows:

$$
\begin{gathered}
\Gamma_{b}=\partial \Omega \cap \partial \Omega_{l o c}, \quad \text { (internal boundary), } \\
\Gamma_{i}=\partial \Omega_{l o c} \cap \Omega, \quad \text { (interface) }, \\
\Gamma_{\infty}=\partial \Omega \backslash \Gamma_{b} . \quad \text { (farfield boundary). }
\end{gathered}
$$

We denote by $n$ the external unit normal vector to $\partial \Omega$ or $\partial \Omega_{l o c}$.

We will make use of the following notation:

$$
\begin{aligned}
& \|v\|_{0, O}=\|v\|_{L_{2}(O)}, \\
& \|v\|_{s, O}=\|v\|_{H^{s}(O)}, \\
& |v|_{1, O}=\|\nabla v\|_{L_{2}(O)},
\end{aligned}
$$

where $O$ is an open bounded domain of $\mathbb{R}^{n}, L^{2}(O)$ is the usual space of square integrable functions over $O$, with norm $\|\cdot\|_{0, O}$, and $H^{s}(O)$ is the usual Sobolev space of functions with derivatives up to order $s$ square integrable over $O$, with norm $\|\cdot\|_{s, O}$.

Let $v$ be the velocity field inside a given incompressible flow such that

$$
\left\{\begin{array}{l}
\operatorname{div} v=0 \text { in } \Omega, \\
v \cdot n=0 \text { on } \Gamma_{b} .
\end{array}\right.
$$

We consider the following convection-diffusion model problem:

Find $\varphi$, a real valued function, defined on $\Omega$ and satisfying

$$
\left\{\begin{aligned}
\operatorname{div}(v \varphi)-\nu \Delta \varphi & =0 \text { in } \Omega, \\
\varphi & =\varphi^{\infty} \text { on } \Gamma_{\infty}, \\
\varphi & =0 \text { on } \Gamma_{b} .
\end{aligned}\right.
$$

Here $v$ is the flow velocity and $\nu$ is the diffusion coefficient. Problems of this form typically occur in fluid mechanics, gas dynamics or wave propagation. 


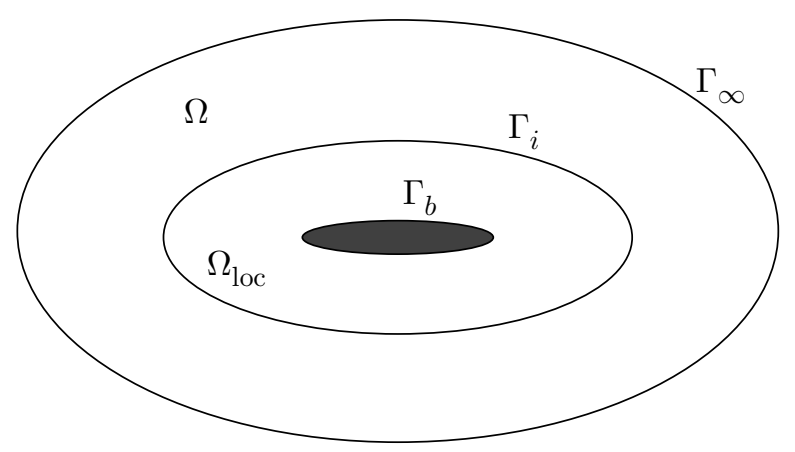

FiguRE 1. Description of the computational domain

Most CFD algorithms will in fact consider the solution of this problem as the stationary solution of the evolution problem (3) described below:

Find $\phi: \Omega \times(0, T) \rightarrow \mathbb{R}$ such that

$$
\left\{\begin{aligned}
\frac{\partial \phi}{\partial t}+\operatorname{div}(v \phi)-\nu \Delta \phi & =0 \text { in } \Omega \times(0, T), \\
\phi & =\phi^{\infty} \text { on } \Gamma_{\infty} \times(0, T), \\
\phi & =0 \text { on } \Gamma_{b} \times(0, T), \\
\phi(0) & =\phi_{0} \text { in } \Omega .
\end{aligned}\right.
$$

The general method then consists in integrating (3) with respect to time until reaching a stationary solution.

\section{General ALgOrithm}

3.1. Time Continuous Case. Let us introduce the local subdomain $\Omega_{l o c}$ (see Figure 1) which has an external boundary $\Gamma_{i}$, and let us consider the trace $\phi_{l o c}$ of $\phi$ on the subdomain $\Omega_{l o c}$ as an independent variable to which we associate an arbitrary independent initial value $\phi_{o l} \neq\left.\phi_{0}\right|_{\Omega_{l o c}}$. We then replace the evolution problem (3) by the following evolution systems:

Find $\phi\left(\right.$ resp. $\left.\phi_{l o c}\right): \Omega \rightarrow \mathbb{R}\left(\right.$ resp. $\left.\Omega_{l o c} \rightarrow \mathbb{R}\right)$ satisfying

$$
\begin{gathered}
\left\{\begin{aligned}
\frac{\partial \phi}{\partial t}+\operatorname{div}(v \phi)-\nu \Delta \phi & =0 \text { in } \Omega \times(0, T), \\
\phi & =\phi^{\infty} \text { on } \Gamma_{\infty} \times(0, T), \\
\nu \frac{\partial \phi}{\partial n} & =\nu \frac{\partial \phi_{l o c}}{\partial n} \text { on } \Gamma_{b} \times(0, T),
\end{aligned}\right. \\
\left\{\begin{aligned}
\frac{\partial \phi_{l o c}}{\partial t}+\operatorname{div}\left(v \phi_{l o c}\right)-\nu \Delta \phi_{l o c} & =0 \text { in } \Omega_{l o c} \times(0, T), \\
\phi_{l o c} & =0 \text { on } \Gamma_{b} \times(0, T), \\
\phi_{l o c} & =\phi \text { on } \Gamma_{i} \times(0, T),
\end{aligned}\right.
\end{gathered}
$$

$$
\phi(0)=\phi_{0} \text { in } \Omega, \phi_{l o c}(0)=\phi_{o l} \text { in } \Omega_{l o c} .
$$


Remark 3.1. The global problem (4) with the initial condition (6) has no no-slip boundary condition. This suppresses the boundary layer which appears at low viscosity and facilitates the numerical solution of this problem. The boundary layers are modeled by the local problems (5)-(6), which are only to be solved on a small domain $\Omega_{l o c}$, with a very fine discretization if needed. The two problems are only coupled by their boundary conditions.

3.2. Time Discrete Case. The general algorithm that we propose for the solution of our model problem (2) is to integrate the evolution problem (4)-(5)-(6) with respect to time until we reach a stationary solution. This integration in time is then achieved by the following uncoupled semi-explicit algorithm, where the operators are treated implicitly inside each subdomain and where one of the coupling boundary conditions is treated explicitly and the other is treated implicitly:

- set $\phi_{l o c}^{0}=\phi_{o l}$ and $\phi^{0}=\phi_{0}$,

- then, for $n \geq 0, \phi_{l o c}^{n}$ and $\phi^{n}$ being known, solve successively

$$
\left\{\begin{aligned}
\frac{\phi_{l o c}^{n+1}-\phi_{l o c}^{n}}{\Delta t}+\operatorname{div}\left(v \phi_{l o c}^{n+1}\right)-\nu \Delta \phi_{l o c}^{n+1} & =0 \text { in } \Omega_{l o c}, \\
\phi_{l o c}^{n+1} & =\phi^{n} \text { on } \Gamma_{i}, \\
\phi_{l o c}^{n+1} & =0 \text { on } \Gamma_{b},
\end{aligned}\right.
$$

$$
\left\{\begin{aligned}
\frac{\phi^{n+1}-\phi^{n}}{\Delta t}+\operatorname{div}\left(v \phi^{n+1}\right)-\nu \Delta \phi^{n+1} & =0 \text { in } \Omega, \\
\phi^{n+1} & =\phi^{\infty} \text { on } \Gamma_{\infty}, \\
\nu \frac{\partial \phi^{n+1}}{\partial n} & =\nu \frac{\partial \phi_{l o c}^{n+1}}{\partial n} \text { on } \Gamma_{b} .
\end{aligned}\right.
$$

Remark 3.2. We have a full uncoupling between (7) and (8). They can (and actually will) be discretized and solved by two independent solution techniques.

Remark 3.3. The fully implicit version of this method consists in replacing the condition

by the condition

$$
\phi_{l o c}^{n+1}=\phi^{n} \text { on } \Gamma_{i}
$$

$$
\phi_{l o c}^{n+1}=\phi^{n+1} \text { on } \Gamma_{i} .
$$

The two subproblems are then coupled at each time step.

Remark 3.4. If in (8) we replace $\Omega$ by $\Omega_{E}$ defined as

$$
\Omega_{E}=\Omega \backslash \Omega_{l o c},
$$

and $\Gamma_{b}$ by $\Gamma_{i}$, and if we set $\Delta t=\infty$, then we obtain a nonoverlapping version of our strategy, which is a standard Dirichlet-Neumann algorithm [14], [15] and therefore requires a relaxation strategy to converge.

Remark 3.5. The initial condition $\phi_{o l}$ is not assumed to be equal to $\phi_{0}$ on the local subdomain $\Omega_{l o c}$, because in most cases it is impossible to impose this condition at the discrete level since the grid used on $\Omega_{l o c}$ will in general be different from the grid used on $\Omega$. In addition, even if we assume $\phi_{o l}=\phi_{0}$, we will not have $\phi_{l o c}^{n}=\phi^{n}$ on $\Omega_{l o c}$ unless we use the fully implicit algorithm on compatible grids. 
In the next section, we describe some applications of the above algorithm to real world problems.

\section{Applications to fluid mechanics and kinetic theory}

4.1. Coupling Navier-Stokes equations. Let us consider the compressible Navier-Stokes equations, which we formally write either as

$$
\frac{\partial W}{\partial t}+\operatorname{div}[F(W)]=0 \quad \text { on } \quad \Omega(\text { conservative form })
$$

or as

$$
\frac{\partial U}{\partial t}+T(U)+D(U)=0 \quad \text { on } \quad \Omega \text { (nonconservative form) }
$$

with $W=(\rho, \rho v, \rho E)$ and $U=(\rho, v, \theta)$ the conservative and nonconservative variables, $F=F_{C}+F_{D}$ the total flux (convective and viscous part), $T$ and $D$ the convective and viscous terms in the nonconservative writing of the Navier-Stokes equations. The problem consists in computing a steady solution of these equations with the boundary conditions

$$
\begin{aligned}
& \rho v, \rho E \text { given on } \Gamma_{\infty} \text { (exterior limit of the domain), } \\
& \rho \text { given on } \Gamma_{\infty} \cap\{x, v(x) \cdot n \leq 0\} \text { (inflow), } \\
& v=0 \text { on the body } \Gamma_{o} \text { (no-slip), } \\
& \theta=\theta_{o} \text { on the body } \Gamma_{o} .
\end{aligned}
$$

The global numerical treatment of these equations faces the following difficulties:

(i) In a conservative calculation, the numerical viscosity of the discretization scheme interferes with the physical viscosity and for a mesh of reasonable size leads to an overprediction of the boundary layer. Moreover, no-slip boundary conditions on the body are difficult to handle for many TVD schemes.

(ii) In a nonconservative calculation, the correct calculation of a shock requires locally a very fine grid if we want to satisfy the Rankine Hugoniot conditions.

In this framework our strategy will couple a global conservative scheme defined on the whole domain, which may be approximated using, for example, a finite volume space discretization [17], and a local approximation defined in the neighborhood of the body, which may be approximated using, for example, a mixed finite element approximation of the nonconservative Navier-Stokes equations [2].

The coupling problem corresponds to solving the following systems:

In $\Omega$, we solve the conservative Navier-Stokes equations

$$
\begin{gathered}
\frac{\partial W}{\partial t}+\operatorname{div}[F(W)]=0 \text { in } \Omega, \\
F(W) \cdot n=\left[\begin{array}{c}
0 \\
n \cdot \sigma(W) \cdot n \\
\tau \cdot \sigma\left(U_{l o c}\right) \cdot n \\
-q\left(U_{l o c}\right) \cdot n
\end{array}\right] \quad \text { on the wall, } \\
W=\text { given imposed value on } \Gamma_{\infty} .
\end{gathered}
$$

And in $\Omega_{l o c}$, we solve the nonconservative Navier-Stokes equations

$$
\begin{array}{r}
\frac{\partial U}{\partial t}+T(U)+D(U)=0 \text { in } \Omega \\
U_{l o c}=0 \text { on } \Gamma_{b} \\
U_{l o c}=W \text { on } \Gamma_{i} .
\end{array}
$$


Above, $n \cdot \sigma \cdot n$ and $\tau \cdot \sigma \cdot n$, respectively, denote the normal and the tangential force exerted by the body on the flow, with $n$ the unit normal vector to the wall oriented towards its interior. Notice that in the global conservative problem the matching conditions are of the Neumann type as in (4), while for the local nonconservative problem these matching boundary conditions are of Dirichlet type as in (5). This coupling gives efficient solution to the points (i) and (ii) mentioned at the beginning of this subsection. More detail about this type of coupling can be found in $[18,19$, 20].

4.2. Coupling Boltzmann equation and Navier-Stokes equations. Another important application is the coupling of different models. Such coupling is fundamental for the solution of transitional regimes for which the aerodynamic models break down [11]. In this case the coupling corresponds to solving simultaneously the Bolztmann equation and Navier-Stokes equations. A brief description is given below.

Let us consider the geometry described in Figure 1. Let $f(x, v, t)$ denotes the particle distribution in the Boltzmann region $\Omega_{l o c}$, which is a small region surrounding the body. Let $W=(\rho, \rho u, \rho E)$ be the value of the conservative variable as computed by a Navier-Stokes model in the whole domain $\Omega$. In $\Omega_{l o c}$, we solve the Boltzmann equation [11]

$$
\frac{\partial f}{\partial t}+v \frac{\partial f}{\partial x}=Q(f, f)
$$

with the boundary conditions

$$
\begin{gathered}
f(v)=\rho M_{u, T}(v) \text { on } \Gamma_{i} \text { if } v \cdot n<0, \\
f(v)=k M_{u_{w, T}}(v, I) \text { on } \Gamma_{b} \text { if } v \cdot n<0 .
\end{gathered}
$$

Everywhere in $\Omega$, we solve the Navier-Stokes equations

$$
\frac{\partial W}{\partial t}+\operatorname{div}[F(W)]=0
$$

with the flux boundary conditions

$$
\begin{gathered}
W=W^{\infty} \text { on } \Gamma_{\infty}, \\
F(W) \cdot n=\left[\begin{array}{c}
0 \\
n \cdot \sigma(W) \cdot n \\
\tau \cdot \sigma_{B o l} \cdot n \\
-q_{B o l} \cdot n
\end{array}\right] \quad \text { on } \Gamma_{b} .
\end{gathered}
$$

Here, $\tau \cdot \sigma_{B o l} \cdot n$ and $q_{B o l} \cdot n$ are the total friction fluxes predicted and computed by the Boltzmann model. The coupling from Boltzmann to Navier-Stokes is therefore achieved by imposing these wall fluxes. Conversely, the Navier-Stokes model acts on the Boltzmann solution by imposing the incoming velocity distribution $\rho M_{u, T}(v)$ on the interface $\Gamma_{i}$, where $(\rho, u, T)$ are the density, velocity and temperature locally predicted by the Navier-Stokes model.

For larger Knudsen number the Navier-Stokes equations are no longer valid in the region next to the body. For such a case, the domain of validity of Navier-Stokes equations is $\Omega_{E}=\Omega \backslash \Omega_{l o c}$ and the Boltzmann equation is applied in the domain $\Omega_{l o c}$. The matching boundary conditions are of Neumann type as in (4). More detail about the coupling of Navier-Stokes equations with the Boltzmann equation can be found in $[1,18,19]$. 


\section{Stationary one-Dimensional CASE}

In this section we present a study of the proposed method for the solution of a stationary one-dimensional problem. For $\Delta t=+\infty$, the above algorithm can be written :

- set $\phi_{l o c}^{0}=\phi_{0}$ and $\phi^{0}=\phi_{0}$,

- then, for $n \geq 0, \phi_{l o c}^{n}$ and $\phi^{n}$ being known, solve

$$
\left\{\begin{aligned}
\operatorname{div}\left(v \phi_{l o c}^{n+1}\right)-\nu \Delta \phi_{l o c}^{n+1} & =0 \text { in } \Omega_{l o c}, \\
\phi_{l o c}^{n+1} & =\phi^{n} \text { on } \Gamma_{i}, \\
\phi_{l o c}^{n+1} & =0 \text { on } \Gamma_{b},
\end{aligned}\right.
$$

$$
\left\{\begin{aligned}
\operatorname{div}\left(v \phi^{n+1}\right)-\nu \Delta \phi^{n+1} & =0 \text { in } \Omega, \\
\phi^{n+1} & =\phi^{\infty} \text { on } \Gamma_{\infty}, \\
\nu \frac{\partial \phi^{n+1}}{\partial n} & =\nu \frac{\partial \phi_{l o c}^{n+1}}{\partial n} \text { on } \Gamma_{b} .
\end{aligned}\right.
$$

In one space dimension we take the global domain $\Omega$ to be the interval $] 0,1[$ of $\mathbb{R}$ decomposed into two fully overlapping subdomains $\Omega=] 0,1\left[\right.$ and $\left.\Omega_{l o c}=\right] h_{2}, 1[$ with

$$
0<h_{2}<1
$$

We then consider the following one-dimensional problem:

Find a real valued function $\varphi$, defined on $\Omega$ and satisfying

$$
\left\{\begin{aligned}
v \varphi^{\prime}-\nu \varphi^{\prime \prime} & =0 \quad \text { on } \Omega \\
\varphi(0) & =a \\
\varphi(1) & =b
\end{aligned}\right.
$$

with a constant velocity $v$. In this one-dimensional case, the above algorithm corresponds to

$$
\begin{aligned}
& \left\{\begin{aligned}
v \varphi_{2}^{(n)^{\prime}}-\nu \varphi_{2}^{(n)^{\prime \prime}} & =0 \quad \text { on }] h_{2}, 1[ \\
\varphi_{2}^{(n)}\left(h_{2}\right) & =\varphi_{1}^{(n-1)}\left(h_{2}\right), \\
\varphi_{2}^{(n)}(1) & =b
\end{aligned}\right. \\
& \left\{\begin{aligned}
v \varphi_{1}^{(n)^{\prime}}-\nu \varphi_{1}^{(n)^{\prime \prime}} & =0 \text { on }] 0,1[, \\
\varphi_{1}^{(n)}(0) & =a, \\
\varphi_{1}^{(n)^{\prime}}(1) & =\varphi_{2}^{(n)^{\prime}}(1) .
\end{aligned}\right.
\end{aligned}
$$


By introducing two relaxation parameters $\theta_{1}$ and $\theta_{2}$, we can also introduce the following variant of the above algorithm:

$$
\begin{aligned}
& \left\{\begin{aligned}
v \varphi_{2}^{(n)^{\prime}}-\nu \varphi_{2}^{(n)^{\prime \prime}} & =0 \text { on }] h_{2}, 1[, \\
\varphi_{2}^{(n)}(1) & =b, \\
\varphi_{2}^{(n)}\left(h_{2}\right) & =\theta_{2} \varphi_{1}^{(n-1)}\left(h_{2}\right)+\left(1-\theta_{2}\right) \varphi_{2}^{(n-1)}\left(h_{2}\right),
\end{aligned}\right. \\
& \left\{\begin{aligned}
v \varphi_{1}^{(n)^{\prime}}-\nu \varphi_{1}^{(n)^{\prime \prime}} & =0 \text { on }] 0,1[ \\
\varphi_{1}^{(n)}(0) & =a, \\
\varphi_{1}^{(n)^{\prime}}(1) & =\theta_{1} \varphi_{2}^{(n)^{\prime}}(1)+\left(1-\theta_{1}\right) \varphi_{1}^{(n-1)^{\prime}}(1) .
\end{aligned}\right.
\end{aligned}
$$

We shall now exhibit the conditions under which the algorithm (15)-(16) converges, and those for which this convergence is optimal. For this purpose, we write the interface solution in the form

$$
\begin{aligned}
& \varphi_{1}^{(n)^{\prime}}(1)=\varphi^{\prime}(1)+\gamma^{n}, \\
& \varphi_{2}^{(n)}\left(h_{2}\right)=\varphi\left(h_{2}\right)+\delta^{n},
\end{aligned}
$$

where $\varphi$ is the solution of the original boundary value problem (12). Using the analytical solutions of the problems (15) and (16), we obtain the following induction formula:

$$
\left(\begin{array}{l}
\delta^{n} \\
\gamma^{n}
\end{array}\right)=M_{I N}\left(\begin{array}{l}
\delta^{(n-1)} \\
\gamma^{(n-1)}
\end{array}\right)
$$

with

$$
M_{I N}=\left(\begin{array}{cc}
1-\theta_{2} & \theta_{2} \frac{\nu}{v} e^{-\left(\frac{v}{\nu}\right)}\left(e^{\frac{v}{\nu} h_{2}}-1\right) \\
\frac{\theta_{1}\left(1-\theta_{2}\right)\left(\frac{v}{\nu}\right)}{e^{\left(\frac{v}{\nu}\right)\left(h_{2}-1\right)}-1} & \frac{e^{\left(\frac{-v}{\nu}\right)} \theta_{1} \theta_{2}\left(e^{\left(\frac{v}{\nu}\right) h_{2}}-1\right)}{\left(e^{\left(\frac{v}{\nu}\right)\left(h_{2}-1\right)}-1\right)}+\left(1-\theta_{1}\right)
\end{array}\right)
$$

This iterative process converges if the spectral radius of the matrix $M_{I N}$ is less than 1. A direct but tedious calculation then yields

Lemma 5.1. The spectral radius of the transfer matrix of the algorithm (15)-(16) is

$$
\rho\left(M_{I N}\right)=\max \left[\frac{1}{2}\left|D \pm \sqrt{D^{2}-4 R}\right|\right]
$$

with

$$
\begin{gathered}
D=2-\left(\theta_{1}+\theta_{2}\right)+\theta_{1} \theta_{2} e^{(-v / \nu)}\left(e^{(v / \nu) h_{2}}-1\right) \frac{1}{e^{-v / \nu} e^{v\left(h_{2} / \nu\right)}-1}, \\
R=\left(1-\theta_{1}\right)\left(1-\theta_{2}\right) .
\end{gathered}
$$

From this calculation we obtain the following results:

i): When $h_{2}$ goes to 1 (nonoverlapping), $D$ goes to $+\infty$, and then $\rho\left(M_{I N}\right)$ goes to $+\infty$. There is no convergence at this limit. 
ii): The optimal convergence is obtained in the case where all the eigenvalues of the matrix $M_{I N}$ are zero, i.e. when $D=0$ and $R=0$. The latter conditions imply in particular

$$
\theta_{1}=1 \text { or } \theta_{2}=1 \text {. }
$$

If we choose, in addition, $\theta_{1}=\theta_{2}$, the condition $D=0$ implies $h_{2}=0$. In this case the subdomain $\Omega_{l o c}$ is equal to the whole domain, and the associated algorithm is no longer of interest.

iii): The convergence of the method depends symmetrically on both relaxation parameters.

According to ii) it is reasonable to take one of the $\theta_{i}$ equal to 1 and call the other $\theta$.

By setting

$$
A=1-\frac{e^{(-v / \nu)}\left(e^{(v / \nu) h_{2}}-1\right)}{e^{(v / \nu)\left(h_{2}-1\right)}-1}
$$

we then have

$$
\rho\left(M_{I N}\right)=|1-\theta A|
$$

In this case, setting

$$
\theta_{\text {opt }}=\left\{1-\frac{e^{(-v / \nu)}\left(e^{(v / \nu) h_{2}}-1\right)}{e^{(v / \nu)\left(h_{2}-1\right)}-1}\right\}^{-1},
$$

which is $<1$, we get the following convergence results:

Theorem 5.1. 1) The convergence is optimal (convergence in 1 iteration) if

$$
\theta=\theta_{\text {opt }} \text {. }
$$

2) The algorithm converges for all $\theta$ in $] 0, \frac{2}{A}[$.

Corollary 5.1. 1) The case without relaxation $(\theta=1)$ converges if and only if

$$
\frac{2}{A} \geq 1
$$

i.e., by setting $d=1-h_{2}$ (overlapping length), if and only if

$$
d \geq \frac{\nu}{v} \log \frac{2}{\left(1+e^{-v / \nu}\right)} \quad \text { (stability condition). }
$$

2) When $v$ goes to zero, we must have $d \geq \frac{1}{2}$.

Remark 5.1. This theorem states that the application of the algorithm (15)-(16) to the time-independent problem (12) converges if and only if the overlap $d$ is sufficiently large. In the same situation, we will see that if the problem (12) can be regarded as the steady solution of a time-dependent problem and we apply our strategy to this evolution problem, the resulting algorithm will converge to the same steady solution but with fewer restrictions on $d$. This motivates the introduction of the time marching algorithm of section 3. Moreover, this time marching technique is well adapted to nonlinear problems such as those encountered in fluid mechanics and kinetic theory (see [1], [18], [19], and [20]). 


\section{IMPLICIT TIME DISCRETIZATION}

6.1. The general algorithm. This section deals with the convergence analysis of the proposed algorithm in multiple dimensions when one uses the fully implicit version of our strategy (4)-(6):

- Set $\phi_{l o c}^{0}=\phi_{o l}$ and $\phi^{0}=\phi_{0}$

- then, for $n \geq 0, \phi_{l o c}^{n}$ and $\phi^{n}$ being known, solve

$$
\begin{aligned}
& \left\{\begin{aligned}
\frac{\phi^{n+1}-\phi^{n}}{\Delta t}+\operatorname{div}\left(v \phi^{n+1}\right)-\nu \Delta \phi^{n+1} & =0 \text { in } \Omega, \\
\phi^{n+1} & =\phi_{\infty} \text { on } \Gamma_{\infty}, \\
\nu \frac{\partial \phi^{n+1}}{\partial n} & =\nu \frac{\partial \phi_{l o c}^{n+1}}{\partial n} \text { on } \Gamma_{b},
\end{aligned}\right. \\
& \left\{\begin{aligned}
\frac{\phi_{l o c}^{n+1}-\phi_{l o c}^{n}}{\Delta t}+\operatorname{div}\left(v \phi_{l o c}^{n+1}\right)-\nu \Delta \phi_{l o c}^{n+1} & =0 \text { in } \Omega_{l o c}, \\
\phi_{l o c}^{n+1} & =\phi^{n+1} \text { on } \Gamma_{i}, \\
\phi_{l o c}^{n+1} & =0 \text { on } \Gamma_{b} .
\end{aligned}\right.
\end{aligned}
$$

6.2. Convergence analysis. Before establishing the convergence result we shall state the preliminary results that will play central roles in the proof of the convergence of our algorithm. The first result states the basic $L^{2}$ and $H^{1}$ local estimates.

Lemma 6.1. We have the following estimates:

$$
\begin{aligned}
\frac{1}{2 \Delta t}\left\|\phi^{n+1}-\phi_{l o c}^{n+1}\right\|_{0, \Omega_{l o c}}^{2} & +\nu\left|\phi^{n+1}-\phi_{l o c}^{n+1}\right|_{1, \Omega_{l o c}}^{2} \\
& \leq \frac{1}{2 \Delta t}\left\|\phi^{n}-\phi_{l o c}^{n}\right\|_{0, \Omega_{l o c}}^{2}, \\
\left\|\phi^{n+1}-\phi_{l o c}^{n+1}\right\|_{0, \Omega_{l o c}}^{2} & \leq \frac{1}{1+2 \nu \Delta t c}\left\|\phi^{n}-\phi_{l o c}^{n}\right\|_{0, \Omega_{l o c}}^{2}, \\
\left\|\phi^{n+1}-\phi_{l o c}^{n+1}\right\|_{0, \Omega_{l o c}}^{2} & \leq\left(\frac{1}{1+2 \nu \Delta t c}\right)^{n+1}\left\|\phi^{0}-\phi_{l o c}^{0}\right\|_{0, \Omega_{l o c}}^{2}, \\
\left\|\phi^{n+1}-\phi_{l o c}^{n+1}\right\|_{0, \Omega_{l o c}}^{2} & +2 \nu \Delta t \sum_{i=p}^{n}\left|\phi^{i+1}-\phi_{l o c}^{i+1}\right|_{1, \Omega_{l o c}}^{2} \\
& \leq\left\|\phi^{p}-\phi_{l o c}^{p}\right\|_{0, \Omega_{l o c}}^{2} \forall p \leq n,
\end{aligned}
$$

where $c$ is the Poincaré constant on the subdomain $\Omega_{l o c}$.

Proof of Lemma 6.1. Subtracting (29) from (28), multiplying the result by $\phi^{n+1}-$ $\phi_{l o c}^{n+1}$ and integrating by parts over $\Omega_{l o c}$, we obtain the following classical relation:

$$
\begin{array}{r}
\int_{\Omega_{l o c}} \frac{1}{\Delta t}\left(\phi^{n+1}-\phi_{l o c}^{n+1}\right)^{2}-\int_{\Omega_{l o c}} \frac{1}{\Delta t}\left(\phi^{n}-\phi_{l o c}^{n}\right)\left(\phi^{n+1}-\phi_{l o c}^{n+1}\right) \\
+\int_{\Omega_{l o c}} \nu\left|\nabla\left(\phi^{n+1}-\phi_{l o c}^{n+1}\right)\right|^{2}=0 .
\end{array}
$$


By using the Cauchy-Schwarz inequality, we obtain the estimate (30). The second estimate (31) follows by using the Poincaré inequality with $c$ the Poincaré constant bounding the squared $H^{1}$ seminorm of any function $v$ of $H^{1}\left(\Omega_{l o c}\right)$ with zero trace on $\Gamma_{i}$ by its squared $L^{2}$ norm. By induction we also obtain the basic $L^{2}$ estimate (32). And finally, we obtain the estimate (33) by summing (30).

The above lemma states that the restriction of $\phi^{n+1}-\phi_{l o c}^{n+1}$ to $\Omega_{l o c}$ converges to 0 in both $L^{2}$ and $H^{1}$ norms. We now establish other $L^{2}$ and $H^{1}$ local estimates. Let $\delta x^{n}$ be defined by

$$
\delta x^{n}=\frac{\left(\phi^{n+1}-\phi_{l o c}^{n+1}\right)-\left(\phi^{n}-\phi_{l o c}^{n}\right)}{\Delta t},
$$

and let $G$ be defined by

$$
G(n)=\frac{\|v\|_{\infty}^{2}}{2 \nu^{2}}\left\|\phi^{n}-\phi_{l o c}^{n}\right\|_{0, \Omega_{l o c}}^{2}+\left|\phi^{n}-\phi_{l o c}^{n}\right|_{1, \Omega_{l o c}}^{2} .
$$

Lemma 6.2. We have the following estimates:

$$
\begin{aligned}
\left\|\delta x^{n}\right\|_{0, \Omega_{l o c}}^{2} & \leq \frac{\nu}{\Delta t}(G(n)-G(n+1)), \\
G(n+1) & \leq\left(\frac{1}{2 \nu \Delta t}+\frac{\|v\|_{\infty}^{2}}{2 \nu^{2}}\right)\left\|\phi^{p-1}-\phi_{l o c}^{p-1}\right\|_{0, \Omega_{l o c}}^{2} \forall p \leq n .
\end{aligned}
$$

Proof of Lemma 6.2. Subtracting the first two equations in (28) and (29), multiplying the result by $\delta x^{n}$ and integrating over $\Omega_{l o c}$, we obtain

$$
\begin{aligned}
0= & \int_{\Omega_{l o c}}\left|\delta x^{n}\right|^{2}+\int_{\Omega_{l o c}} \operatorname{div}\left(v\left(\phi^{n+1}-\phi_{l o c}^{n+1}\right)\right) \delta x^{n} \\
& +\nu \int_{\Omega_{l o c}} \nabla\left(\phi^{n+1}-\phi_{l o c}^{n+1}\right) \nabla \delta x^{n} \\
& -\nu \int_{\partial \Omega_{l o c}} \frac{\partial}{\partial n}\left(\phi^{n+1}-\phi_{l o c}^{n+1}\right) \delta x^{n} .
\end{aligned}
$$

Using the boundary conditions in (28) and (29) and the Cauchy-Schwarz inequality, we obtain

$$
\begin{aligned}
\left\|\delta x^{n}\right\|_{0, \Omega_{l o c}}^{2} \leq & \frac{1}{2}\|v\|_{\infty}^{2}\left|\phi^{n+1}-\phi_{l o c}^{n+1}\right|_{1, \Omega_{l o c}}^{2}+\frac{1}{2}\left\|\delta x^{n}\right\|_{0, \Omega_{l o c}}^{2} \\
& +\frac{\nu}{2 \Delta t}\left|\phi^{n}-\phi_{l o c}^{n}\right|_{1, \Omega_{l o c}}^{2}-\frac{\nu}{2 \Delta t}\left|\phi^{n+1}-\phi_{l o c}^{n+1}\right|_{1, \Omega_{l o c}}^{2} .
\end{aligned}
$$

Now the relation (30) (Lemma 6.1) leads to the first estimate of our lemma. In fact, this estimate implies that $G$ is a decreasing function. This property then yields

$$
\begin{aligned}
(n+2-p) G(n+1) & \leq \sum_{i=p}^{n+1} G(i) \\
& \leq \sum_{i=p}^{n+1}\left|\phi^{i}-\phi_{l o c}^{i}\right|_{1, \Omega_{l o c}}^{2}+\frac{\|v\|_{\infty}^{2}}{2 \nu^{2}} \sum_{i=p}^{n+1}\left\|\phi^{i}-\phi_{l o c}^{i}\right\|_{0, \Omega_{l o c}}^{2} .
\end{aligned}
$$

Using again the relations (30) and (31) (Lemma 6.1) yields the second estimate (38). And the lemma is proved. 
Next we establish the global $L^{2}$ and $H^{1}$ estimates. Let $\bar{\phi}^{n+1}$ be defined as follows:

$$
\bar{\phi}^{n+1}=\left\{\begin{array}{lll}
\phi^{n+1}-\varphi & \text { in } & \Omega \backslash \Omega_{l o c} \\
\phi_{l o c}^{n+1}-\varphi & \text { in } & \Omega_{l o c}
\end{array}\right.
$$

where $\varphi$ is the solution of the stationary problem (2). By construction, $\bar{\phi}^{n+1}$ satisfies the following equations:

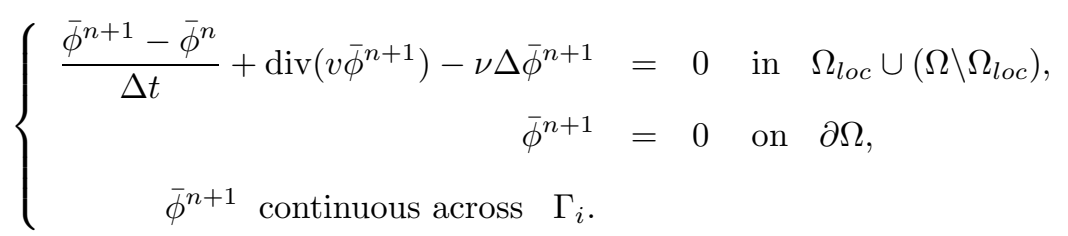

Let $A, B_{1}$, and $B_{2}$ be defined as follows:

$$
\begin{gathered}
A=\frac{1}{1+\left(\nu c-2 c_{1}^{2}\right) \Delta t}, \\
B_{1}=\frac{\Delta t}{c_{1}^{2}} A, \\
B_{2}=\Delta t\left(\frac{\|v\|_{\infty}^{2}}{c_{1}^{2}}+\nu\right) A,
\end{gathered}
$$

where $c$ is the Poincaré constant and $c_{1}>0$ is an arbitrary constant. We have the following lemma.

Lemma 6.3. We have the following estimates:

$$
\begin{gathered}
\left(\frac{1}{2 \Delta t}-c_{1}^{2}\right)\left\|\bar{\phi}^{n+1}\right\|_{0, \Omega}^{2}+\frac{\nu}{2}\left|\bar{\phi}^{n+1}\right|_{1, \Omega}^{2} \\
\leq \frac{1}{2 \Delta t}\left\|\bar{\phi}^{n}\right\|_{0, \Omega}^{2}+\frac{1}{2 c_{1}^{2}}\left\|\delta x^{n}\right\|_{0, \Omega_{l o c}}^{2} \\
+\left(\frac{\|v\|_{\infty}^{2}}{2 c_{1}^{2}}+\frac{\nu}{2}\right)\left|\phi_{l o c}^{n+1}-\phi^{n+1}\right|_{1, \Omega_{l o c}}^{2} \\
\left\|\bar{\phi}^{n+1}\right\|_{0, \Omega}^{2} \leq A\left\|\bar{\phi}^{n}\right\|_{0, \Omega}^{2}+B_{1}\left\|\delta x^{n}\right\|_{0, \Omega_{l o c}}^{2}+B_{2}\left|\phi_{l o c}^{n+1}-\phi^{n+1}\right|_{1, \Omega_{l o c}}^{2} .
\end{gathered}
$$

Proof of Lemma 6.3. Multiplying the equation (42) by $\bar{\phi}^{n+1}$, integrating by parts over $\Omega_{l o c}$ and $\Omega \backslash \Omega_{l o c}$ and taking into account the boundary conditions in (42), we obtain the following relation:

$$
\begin{gathered}
\int_{\Omega} \frac{\bar{\phi}^{n+1}-\bar{\phi}^{n}}{\Delta t} \bar{\phi}^{n+1}+\nu \int_{\Omega}\left|\nabla \bar{\phi}^{n+1}\right|^{2} \\
-\nu \int_{\Gamma_{i}} \frac{\partial}{\partial n}\left(\phi_{l o c}^{n+1}-\phi^{n+1}\right) \bar{\phi}^{n+1}=0 .
\end{gathered}
$$

On $\Omega_{l o c}, \phi_{l o c}^{n+1}-\phi^{n+1}$ satisfies the following equation:

$$
\frac{\left(\phi_{l o c}^{n+1}-\phi^{n+1}\right)-\left(\phi_{l o c}^{n}-\phi^{n}\right)}{\Delta t}+\operatorname{div}\left[v\left(\phi_{l o c}^{n+1}-\phi^{n+1}\right)\right]-\nu \Delta\left(\phi_{l o c}^{n+1}-\phi^{n+1}\right)=0 .
$$


Therefore, multiplying the above equation by $\bar{\phi}^{n+1}$, integrating by parts and using the Cauchy-Schwarz inequality, we obtain

$$
\begin{aligned}
\nu \mid \int_{\Gamma_{i}} & \frac{\partial}{\partial n}\left(\phi_{l o c}^{n+1}-\phi^{n+1}\right) \bar{\phi}^{n+1} \mid \\
\leq & \frac{1}{2 c_{1}^{2}}\left\|\delta x^{n}\right\|_{0, \Omega_{l o c}}^{2}+\frac{1}{2} c_{1}^{2}\left\|\bar{\phi}^{n+1}\right\|_{0, \Omega_{l o c}}^{2}+\frac{\|v\|_{\infty}^{2}}{2 c_{1}^{2}}\left|\phi_{l o c}^{n+1}-\phi^{n+1}\right|_{1, \Omega_{l o c}}^{2} \\
& +\frac{1}{2} c_{1}^{2}\left\|\bar{\phi}^{n+1}\right\|_{0, \Omega_{l o c}}^{2}+\frac{\nu}{2}\left|\phi_{l o c}^{n+1}-\phi^{n+1}\right|_{1, \Omega_{l o c}}^{2}+\frac{\nu}{2}\left|\bar{\phi}^{n+1}\right|_{1, \Omega_{l o c}}^{2},
\end{aligned}
$$

with $c_{1}>0$ arbitrary. Combining the above inequality with (45), bounding the local norm $|f|_{i, \Omega_{l o c}}$ by $|f|_{i, \Omega}$ and using the Cauchy-Schwarz inequality, we obtain the estimate (43). The estimate (44) results immediately from the estimate (43) by applying Poincaré inequality on $\Omega$ with $c$ the Poincaré constant. The lemma is proved.

Finally, we are in a position to state the main result of this section.

Theorem 6.1. The solution of the algorithm (28)-(29) converges linearly in $H^{1}(\Omega)$ to the solution of the stationary problem (2) for all values of $\Delta t$ and all choices of $\Omega_{l o c}$.

Proof of Theorem 6.1. Let $c_{1}$ be chosen such that $\nu c-2 c_{1}^{2}>0$. Using relation (44) (Lemma 6.3), we obtain by induction

$$
\begin{aligned}
\left\|\bar{\phi}^{n+1}\right\|_{0, \Omega}^{2} \leq & A^{p}\left\|\bar{\phi}^{n+1-p}\right\|_{0, \Omega}^{2} \\
& +\sum_{i=0}^{p-1} A^{i}\left(B_{1}\left\|\delta x^{n-i}\right\|_{0, \Omega_{l o c}}^{2}+B_{2}\left|\phi_{l o c}^{n+1-i}-\phi^{n+1-i}\right|_{1, \Omega_{l o c}}^{2}\right) .
\end{aligned}
$$

Since $A<1$ by assumption on $c_{1}$, this implies

$$
\begin{aligned}
\left\|\bar{\phi}^{n+1}\right\|_{0, \Omega}^{2} \leq & A^{p}\left\|\bar{\phi}^{n+1-p}\right\|_{0, \Omega}^{2}+B_{1} \sum_{i=n+1-p}^{n}\left\|\delta x^{i}\right\|_{0, \Omega_{l o c}}^{2} \\
& +B_{2} \sum_{i=n+1-p}^{n}\left|\phi_{l o c}^{i+1}-\phi^{i+1}\right|_{1, \Omega_{l o c}}^{2} .
\end{aligned}
$$

Now, using (37) (Lemma 6.2) and (33) (Lemma 6.1), we obtain

$$
\begin{aligned}
\left\|\bar{\phi}^{n+1}\right\|_{0, \Omega}^{2} \leq & A^{p}\left\|\bar{\phi}^{n+1-p}\right\|_{0, \Omega}^{2}+B_{1} \frac{\nu}{\Delta t}(G(n+1-p)-G(n+1)) \\
& +B_{2} \frac{1}{2 \nu \Delta t}\left\|\phi_{l o c}^{n+1-p}-\phi^{n+1-p}\right\|_{0, \Omega_{l o c}}^{2} .
\end{aligned}
$$

The same relation written between 0 and $n+1-p$ yields

$$
\begin{aligned}
\left\|\bar{\phi}^{n+1-p}\right\|_{0, \Omega}^{2} \leq & A^{n+1-p}\left\|\bar{\phi}^{o}\right\|_{0, \Omega_{l o c}}^{2}+B_{1} \frac{\nu}{\Delta t}(G(0)-G(n-p+1)) \\
& +B_{2} \frac{1}{2 \nu \Delta t}\left\|\phi^{0}-\phi_{l o c}^{0}\right\|_{0, \Omega_{l o c}}^{2} .
\end{aligned}
$$


By combining this relation with (49), we finally obtain

$$
\begin{aligned}
\left\|\bar{\phi}^{n+1}\right\|_{0, \Omega}^{2} \leq & A^{n+1}\left\|\bar{\phi}^{0}\right\|_{0, \Omega}^{2} \\
& +B_{1} \frac{\nu}{\Delta t} G(0)+B_{2} \frac{1}{2 \nu \Delta t}\left\|\phi^{0}-\phi_{l o c}^{0}\right\|_{0, \Omega_{l o c}}^{2} \\
& +B_{1} \frac{\nu}{\Delta t} G(n+1-p)+\frac{B_{2}}{2 \nu \Delta t}\left\|\phi_{l o c}^{n+1-p}-\phi^{n+1-p}\right\|_{0, \Omega_{l o c}}^{2} .
\end{aligned}
$$

Choosing $p$ such that $n=2 p+q, q \geq 1$ and using (38) (Lemma 6.2), we conclude that

$$
\left\|\bar{\phi}^{n+1}\right\|_{0, \Omega}^{2} \leq A^{n+1} C_{2}+A^{p} C_{3}+C_{4}\left\|\phi_{l o c}^{p}-\phi^{p}\right\|_{0, \Omega_{l o c}}^{2},
$$

which, from (32) (Lemma 6.1) implies the linear convergence of $\left\|\bar{\phi}^{n+1}\right\|_{0, \Omega}^{2}$ to 0 .

On the other hand, by combining (37) (Lemma 6.2) and (43) (Lemma 6.3) we obtain

$$
\begin{aligned}
\left(\frac{1}{2 \Delta t}-c_{1}^{2}\right)\left\|\bar{\phi}^{n+1}\right\|_{0, \Omega}^{2} & +\frac{\nu}{2}\left|\bar{\phi}^{n+1}\right|_{1, \Omega}^{2} \\
\leq \frac{1}{2 \Delta t}\left\|\bar{\phi}^{n}\right\|_{0, \Omega}^{2} & +\frac{\nu}{2 c_{1}^{2} \Delta t}(G(n)-G(n+1)) \\
& +\left(\frac{\|v\|_{\infty}^{2}}{2 c_{1}^{2}}+\frac{\nu}{2}\right)\left|\phi_{l o c}^{n+1}-\phi^{n+1}\right|_{1, \Omega_{l o c}}^{2}
\end{aligned}
$$

Therefore by using (30) we obtain

$$
\begin{aligned}
\left(\frac{1}{2 \Delta t}-c_{1}^{2}\right)\left\|\bar{\phi}^{n+1}\right\|_{0, \Omega}^{2} & +\frac{\nu}{2}\left|\bar{\phi}^{n+1}\right|_{1, \Omega}^{2} \\
\leq \frac{1}{2 \Delta t}\left\|\bar{\phi}^{n}\right\|_{0, \Omega}^{2} & +\frac{\nu}{2 c_{1}^{2} \Delta t}(G(n)-G(n+1)) \\
& +\left(\frac{\|v\|_{\infty}^{2}}{2 c_{1}^{2}}+\frac{\nu}{2}\right)\left(\frac{\left\|\phi_{l o c}^{n}-\phi^{n}\right\|_{0, \Omega_{l o c}}^{2}}{2 \nu \Delta t}\right) .
\end{aligned}
$$

Our result now follows from (38) (Lemma 6.2), (32) (Lemma 6.1), and the linear convergence of $\left\|\bar{\phi}^{n}\right\|_{0, \Omega}$.

6.3. Convergence of a fixed point method for the implicit scheme. The implicit scheme proposed in this section couples the global and the local problem. To uncouple them, it is advisable to use the fixed point algorithm. In this subsection we just describe the algorithm. For the analysis we refer to [12, 13, 18]. The algorithm is as follows:

- set $\phi_{l o c, 0}^{0}=\psi_{o l}$ and $\phi^{0}=\psi_{0}$,

- then, for $k \geq 0, \phi_{\left.k\right|_{\Gamma_{i}}}^{n+1}$ being known, solve

$$
\left\{\begin{aligned}
\frac{\phi_{l o c, k+1}^{n+1}-\phi_{l o c}^{n}}{\Delta t}+\operatorname{div}\left(v \phi_{l o c, k+1}^{n+1}\right)-\nu \Delta \phi_{l o c, k+1}^{n+1} & =0 \text { in } \Omega_{l o c}, \\
\phi_{l o c, k+1}^{n+1} & =\phi_{k}^{n+1} \text { on } \Gamma_{i}, \\
\phi_{l o c, k+1}^{n+1} & =0 \text { on } \Gamma_{b},
\end{aligned}\right.
$$




$$
\left\{\begin{aligned}
\frac{\phi_{k+1}^{n+1}-\phi^{n}}{\Delta t}+\operatorname{div}\left(v \phi_{k+1}^{n+1}\right)-\nu \Delta \phi_{k+1}^{n+1} & =0 \text { in } \Omega \\
\phi_{k+1}^{n+1} & =\phi_{\infty} \text { on } \Gamma_{\infty}, \\
\nu \partial \phi_{k+1}^{n+1} / \partial n & =\nu \partial \phi_{l o c, k+1}^{n+1} / \partial n \quad \text { on } \Gamma_{b} .
\end{aligned}\right.
$$

We will study now the algorithm (53)-(54). By setting

$$
\begin{aligned}
\psi_{l o c, k, q} & =\phi_{l o c, k+1}^{n+1}-\phi_{l o c, q+1}^{n+1}, \\
\psi_{k, q} & =\left(\phi_{k}^{n+1}-\phi_{q}^{n+1}\right),
\end{aligned}
$$

we observe that $\psi_{l o c, k, q}$ and $\psi_{k, q}$ satisfy the following equations:

$$
\begin{aligned}
& \left\{\begin{aligned}
\psi_{l o c, k, q} / \Delta t+\operatorname{div}\left(v \psi_{l o c, k, q}\right)-\nu \Delta \psi_{l o c, k, q} & =0 \text { in } \Omega_{l o c}, \\
\psi_{l o c, k, q} & =\psi_{k-1, q-1} \text { on } \Gamma_{i}, \\
\psi_{l o c, k, q} & =0 \text { on } \Gamma_{b},
\end{aligned}\right. \\
& \left\{\begin{aligned}
\psi_{k, q} / \Delta t+\operatorname{div}\left(v \psi_{k, q}\right)-\nu \Delta \psi_{k, q} & =0 \text { in } \Omega, \\
\psi_{k, q} & =0 \text { on } \Gamma_{\infty}, \\
\nu \frac{\partial \psi_{k, q}}{\partial n} & =\nu \frac{\partial \psi_{l o c, k, q}}{\partial n} \text { on } \Gamma_{b} .
\end{aligned}\right.
\end{aligned}
$$

If $\Delta t$ is sufficiently small, we prove in [13] that $\psi_{k, q}$ and $\psi_{l o c, k, q}$ converge linearly to zero. Hence the sequences $\phi_{k}^{n+1}$ and $\phi_{l o c, k}^{n+1}$ are Cauchy sequences which converge linearly to the unique solutions $\phi^{n+1}$ and $\phi_{l o c}^{n+1}$ of the implicit scheme. This guarantees the convergence of the above fixed point algorithm.

\section{Numerical analysis of the stability of the Algorithm (7)-(8)}

In this section we focus on the application of the explicit time marching algorithm (7)-(8) studied in the previous sections to the numerical solution of the steady boundary value problem (2). We first assume that the boundary condition on $\Gamma_{b}$ in (8) is explicit:

$$
\nu \frac{\partial \phi^{n+1}}{\partial n}=\nu \frac{\partial \phi_{l o c}^{n}}{\partial n}
$$

so that the resulting algorithm is parallel (Jacobi type).

Here, $\Omega$ denotes the domain surrounding the obstacle (an ellipse in our numerical study), as described in Figure 1. The global and local subdomains are discretized by fully overlapping compatible finite element grids. The global mesh contains 1378 nodes and 2662 elements (see Figure 2). Furthermore, the time marching algorithm is initialized by setting $\phi_{0}$ equal to zero.

In a first step, the velocity field is obtained by solving the following inviscid incompressible flow problem:

$$
\left\{\begin{array}{l}
\operatorname{div} v=0 \\
\operatorname{curl} v=0 \\
v_{\infty}=(1,0) \\
v \cdot n=0 \text { on the body } \Gamma_{b}
\end{array}\right.
$$




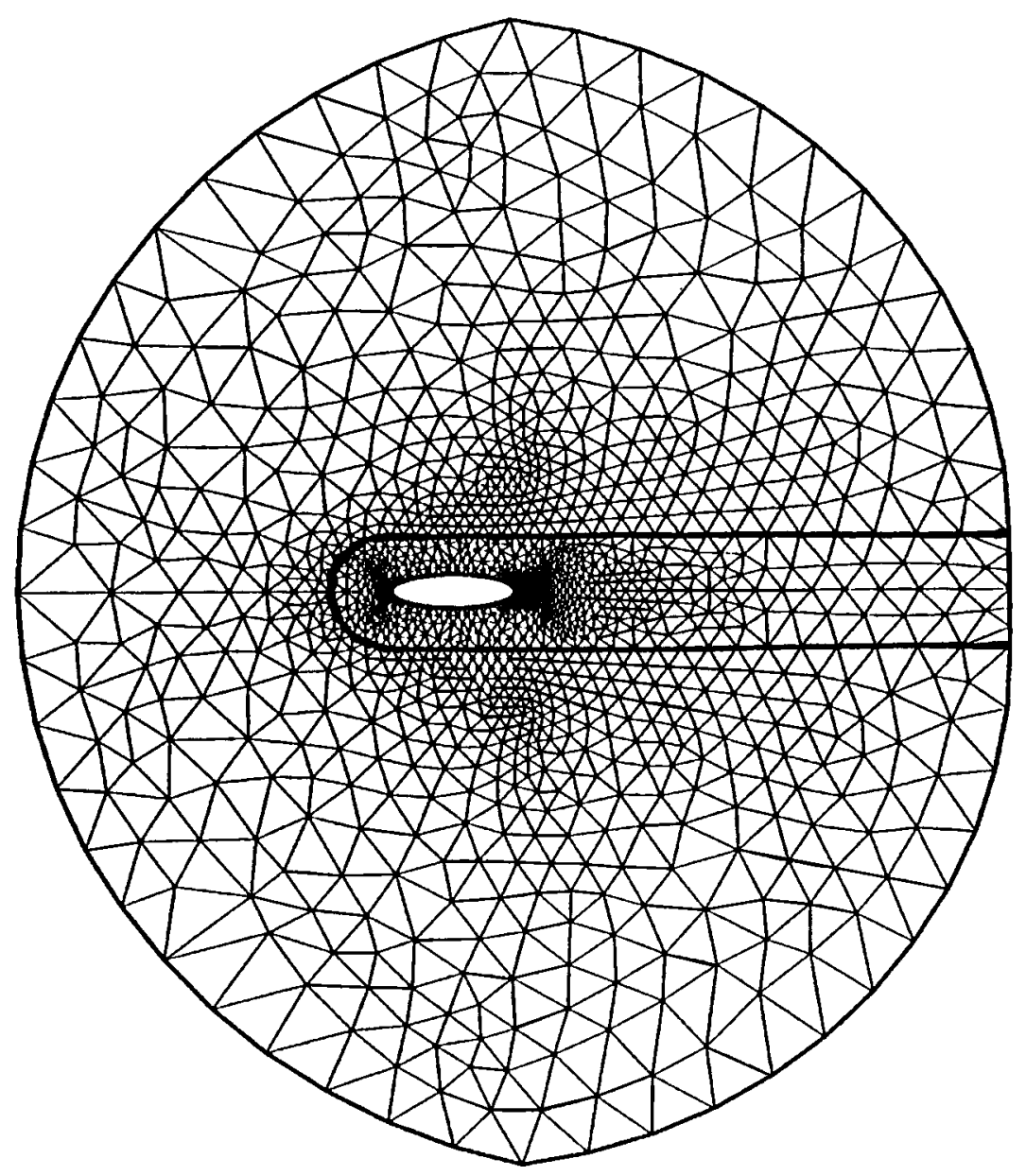

Figure 2. Description of the finite element mesh and of the local subdomain.

with a first order finite element method using the same global mesh.

If we set $v=0$, the algorithm may or may not converge depending on the values of $\nu \Delta t$. More precisely, we observe that the algorithm converges linearly when $\nu \Delta t<\alpha_{0}$, and diverges otherwise. This is graphically shown in Figures 3-7, where the values of $\frac{\left\|\phi^{n+1}-\phi^{n}\right\|}{\Delta t\left\|\phi_{0}\right\|_{\infty}}$ are plotted versus the iteration count $n$ for $\nu \Delta t$ equal respectively to $10^{-6}, 10^{-1}, 1$, and 10 . Further, when the velocity is taken sufficiently large, the algorithm becomes unconditionally stable. In particular, the initialization of our algorithm by $\phi_{0}=0$ with $\left\|v_{\infty}\right\|=1, \nu=0.1$ and $\Delta t=100$ leads to a converging algorithm (Figure 7).

By intuition such a behavior seems natural. An overestimation of the solution $\phi^{n}$ at the interface $\Gamma_{i}$ implies an overestimation of the friction forces on $\Gamma_{b}$. For sufficiently small time steps, this overestimation will not affect the value of $\phi^{n+1}$ on $\Gamma_{i}$ and can therefore be ignored at the next time step. If the Reynolds number is sufficiently large, this error will only affect the wake region but will not have 


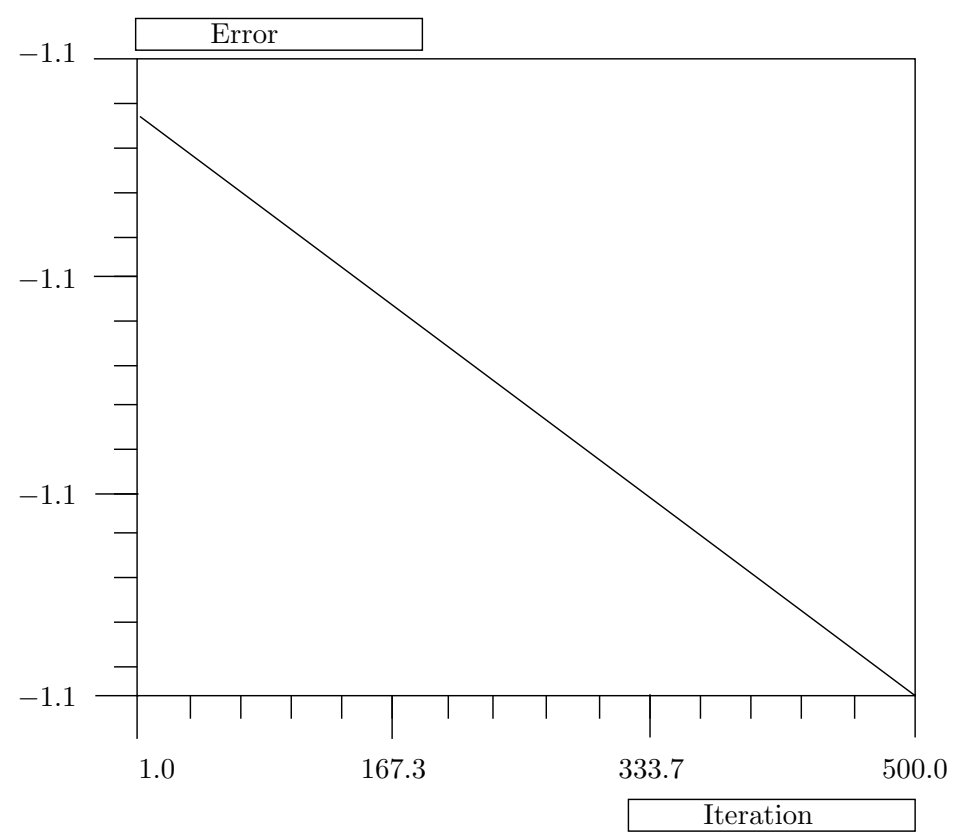

FIGURE 3

any influence at the interface $\Gamma_{i}$. By contrast, for large $\Delta t$ and $\nu$, this error does affect the value of $\phi^{n+1}$ on $\Gamma_{i}$. The influence of the error on $\phi^{n+1}$ may be amplified throughout the iteration process.

Another variant of the algorithm consists of replacing the explicit Dirichlet condition

$$
\phi_{l o c}^{n+1}=\phi^{n} \text { on } \Gamma_{i}
$$

in the algorithm (7)-(8) by the implicit condition

$$
\phi_{l o c}^{n+1}=\phi^{n+1} \text { on } \Gamma_{i} .
$$

In fact, this implies replacing the previously parallel algorithm (Jacobi-like) by the sequential algorithm (Gauss-Seidel-like).

When we solve the pure diffusion problem (i.e. flow velocity $v=0$ ) with $\nu=1$ and $\Delta t=1$ (respectively $\Delta t=2$ ) we obtain a better convergence history:

- The speed of the new algorithm is linear and clearly faster than the parallel algorithm.

- The domain of convergence is moderately larger (see Table 1).

To study the convergence behavior of both algorithms experimentally in more detail we assume that we have a linear behavior of our algorithm, and hence the error at the iteration $n$ will satisfy the following relation:

$$
\left\|\phi^{n+1}-\phi^{n}\right\|_{\infty} \approx K^{n}\left\|\phi^{1}-\phi^{0}\right\|_{\infty} .
$$

The algorithm converges if $K<1$. An estimate for $K$ can be found by considering, as in Table 1, the ratio

$$
-\frac{1}{n} \log \frac{\left\|\phi^{n+1}-\phi^{n}\right\|_{\infty}}{\left\|\phi^{1}-\phi^{0}\right\|_{\infty}}=-\log K,
$$




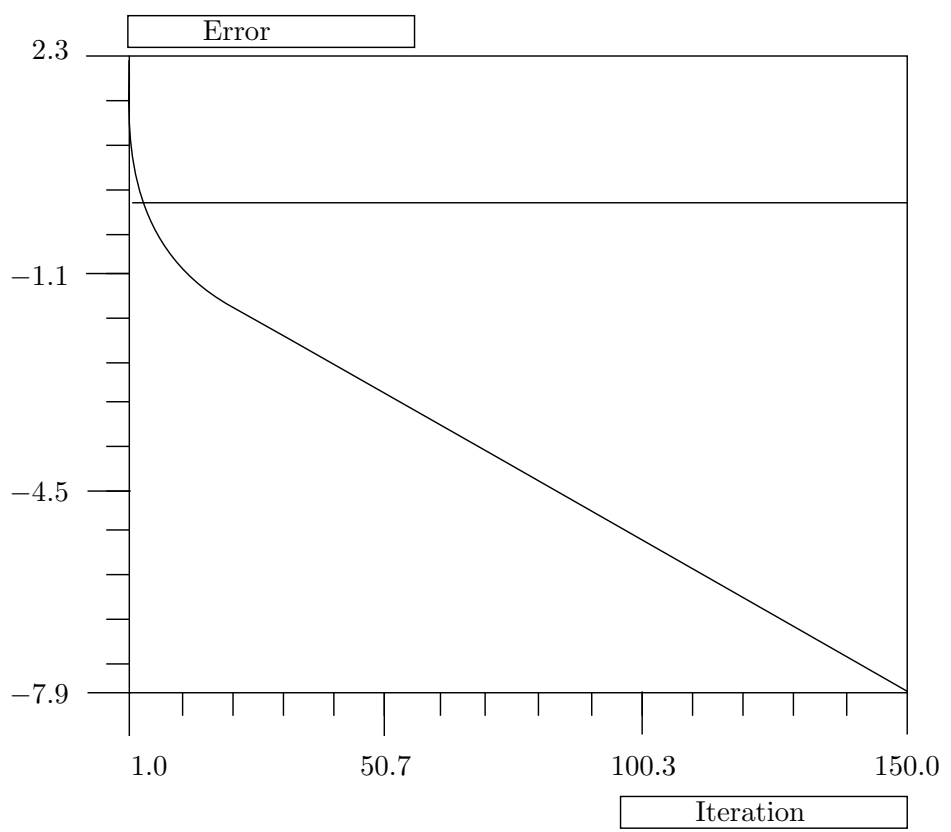

Figure 4

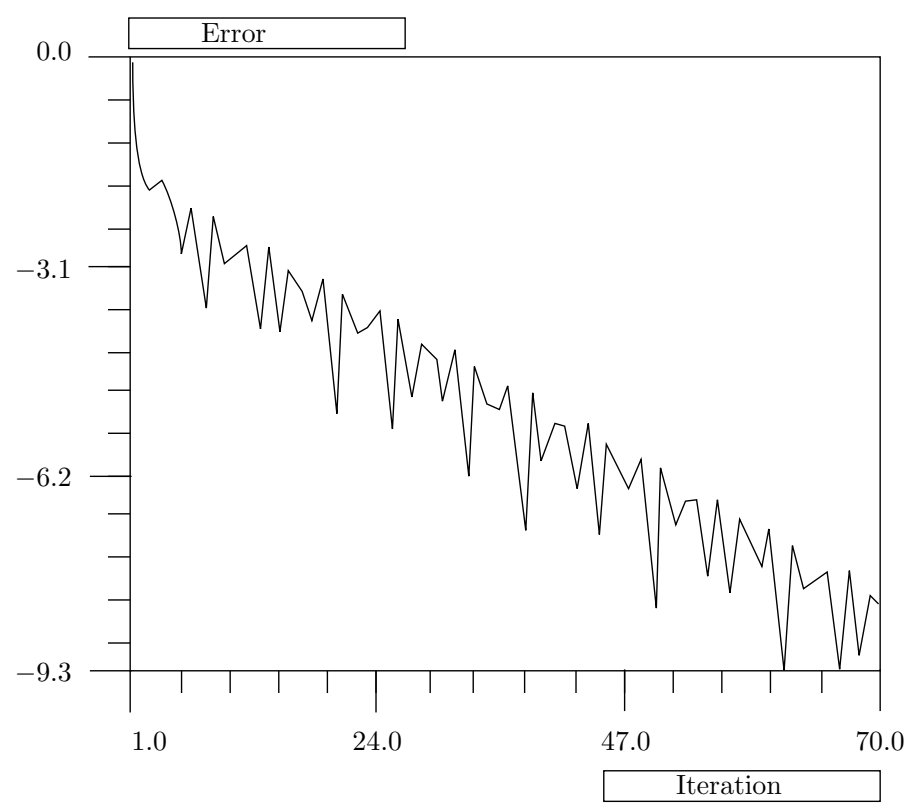

FiguRe 5 


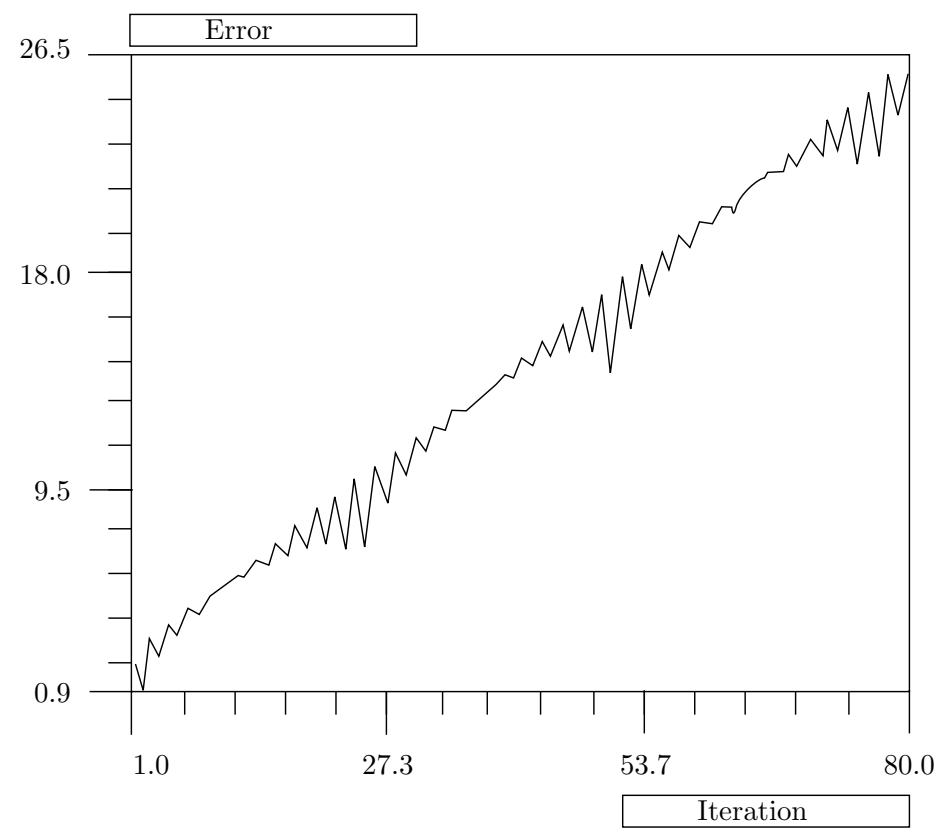

Figure 6

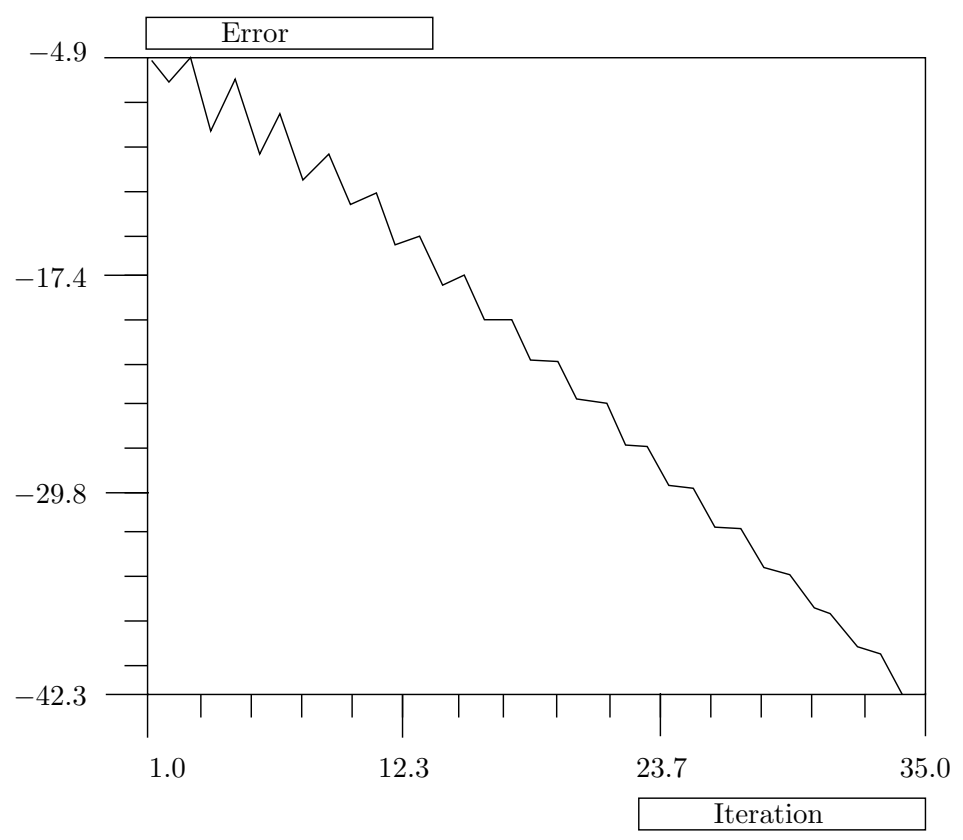

FiguRE 7 
TABLE 1. Contraction constant (in fact minus its logarithm) versus $\nu \Delta t$ for the explicit (Jacobi) and semi-explicit (Gauss-Seidel) version of our coupling algorithm. We observe divergence for $V=0$ and $\nu \Delta t>2$, and convergence otherwise.

\begin{tabular}{|c|c|c|c|c|c|c|c|c|}
\hline$\nu \Delta t$ & $1 / 1000$ & $1 / 10$ & $1 / 2$ & 2 & 5 & 10 & 50 & 1000 \\
\hline $\begin{array}{c}\text { Gauss-Seidel } \\
V=0\end{array}$ & 0.06 & 0.1 & 0.22 & 0.5 & -0.27 & -0.5 & -0.75 & -0.8 \\
\hline $\begin{array}{c}\text { Jacobi } \\
V=0\end{array}$ & - & 0.1 & 0.22 & 0 & -0.09 & -0.25 & -0.4 & -0.41 \\
\hline $\begin{array}{c}\text { Gauss-Seidel } \\
V=10\end{array}$ & 0.03 & 0.25 & 1.46 & 2.12 & 2.8 & 2.6 & 2.4 & 2.4 \\
\hline $\begin{array}{c}\text { Jacobi } \\
V=10\end{array}$ & 0.03 & 0.28 & 1.15 & 1.15 & 1.15 & 1.15 & 1.14 & 1.14 \\
\hline $\begin{array}{c}\text { Jacobi } \\
V=1000\end{array}$ & 0.23 & 2.79 & 2.8 & 2.7 & 2.75 & 2.8 & - & - \\
\hline
\end{tabular}

which is displayed as a function of $(\nu \Delta t)$ for $n=14$ and different values of $V=\frac{v}{\nu}$. A negative value of this ratio means divergence of the algorithm. As expected, this ratio is positive for sufficiently small values of $\Delta t$ and converges to zero as $\Delta t$ goes to zero.

In this table, we observe that for $V=0$ and $\nu \Delta t<\alpha_{0} \approx 2$, the algorithm converges. However the convergence is slow since the minimal contraction constant $K_{\min }$ (for the optimal value of $\nu \Delta t$ ) is close to one (see Table 2). For $V=10$, the algorithm converges for a much larger range of values of $\nu \Delta t$ and the optimal contraction constant is much smaller. This is summarized in Table 2, where we have displayed the best possible contraction constants for each of the coupling algorithms and for different values of the Reynolds number $V=\frac{v}{\nu}$.

TABLE 2. Minimal contraction constant versus the Reynolds number $V$ for both sequential and parallel versions of the algorithm.

\begin{tabular}{|cc|cc|}
\hline \multicolumn{2}{|c|}{ Jacobi } & (parallel) & \multicolumn{2}{|c|}{ Gauss-Seidel (sequential) } \\
\hline \hline$V$ & $K_{\min }$ & \multicolumn{2}{|c|}{$K_{\min }$} \\
\hline 0 & 0.85 & 0 & 0.68 \\
\hline 10 & 0.50 & 10 & 0.11 \\
\hline $10^{3}$ & 0.14 & \multicolumn{2}{c}{} \\
\hline
\end{tabular}

\section{Conclusion}

We have analyzed the convergence properties of a time marching algorithm for solving a domain decomposed advection-diffusion problem with full overlapping and coupling by friction. We were able to prove theoretically the unconditional stability and linear convergence of the fully implicit algorithm $(\S 6)$.

When using the uncoupled semi-explicit algorithm in the general case, numerical evidence indicates that this algorithm is unstable for large values of $\Delta t$ and small overlapping, and that it becomes linearly convergent when $\Delta t$ is below a Reynoldsnumber-dependent threshold $(\S 7)$. This conditional stability is not a real issue for practical CFD problems because most solvers already require the use of small time 
steps inside each domain. Nevertheless, it would be nicer to derive an uncoupled unconditionally stable version of the present time marching algorithm.

\section{REFERENCES}

[1] J.-F. Bourgat, P. Le Tallec and M. D. Tidriri, Coupling Boltzman and Navier-Stokes equations by friction, J. Comp. Phy. 127, 227-245 CMP 98:09 (1996).

[2] M.O. Bristeau, R. Glowinski, L. Dutto, J. Périaux, G. Rogé, Compressible viscous flow calculations using compatible finite element approximations, 7th Int. Conf. on Finite Element Methods in Flow Problems, Huntsville, Alabama (1989) ; et International Journal for Numerical Methods in Fluids 11 (1990), pp. 719-749. MR 91g:76062

[3] R. Glowinski, G. Golub, G. A. Meurant and J. Périaux (eds), Proceedings of the First International Symposium on Domain Decomposition Methods for Partial Differential Equations, Paris, France, January 7-9, 1987, (SIAM, Philadelphia, 1988). MR 89f:65005

[4] T. Chan, R. Glowinski, J. Périaux and O. Widlund (eds), Proceedings of the Second International Symposium on Domain Decomposition Methods for Partial Differential Equations, Los Angeles, California, January 14-16, 1988, (SIAM, Philadelphia, 1989). MR 89j:65010

[5] T. Chan, R. Glowinski, J. Périaux and O. Widlund (eds), Proceedings of the Third International Symposium on Domain Decomposition Methods for Partial Differential Equations, Houston, Texas, March 20-22, 1989, (SIAM, Philadelphia, 1990). MR 91e:65010

[6] R. Glowinski, Y. Kuznetsov, G. Meurant, J. Périaux and O. Widlund (eds), Proceedings of the Fourth International Symposium on Domain Decomposition Methods for Partial Differential Equations, Moscow, May 21-25, 1990, (SIAM, Philadelphia, 1991). MR 92a:65023

[7] T. Chan, D. Keyes, G. Meurant, J. Scroggs and R. Voigt (eds), Proceedings of the Fifth International Symposium on Domain Decomposition Methods for Partial Differential Equations, Norfolk, Virginia, May 6-8, 1991, (SIAM, Philadelphia, 1992). MR 93g:65009

[8] A. Quarteroni (ed), Proceedings of the Sixth International Symposium on Domain Decomposition Methods for Partial Differential Equations, Como, June 15-19, 1992, Contemp. Math. 157 (AMS, Providence, 1994). MR 94i:65004

[9] Y. Achdou and O. Pironneau, A fast solver for Navier-Stokes equations in the laminar regime using mortar finite element and boundary element methods, Technical Report 93-277 (Centre de Mathématiques Appliquées, Ecole Polytechnique, Paris, 1993).

[10] C. Canuto and A. Russo, On the Elliptic-Hyperbolic Coupling. I: The Advection Diffusion Equation via the $\xi$-formulation, Math. Models and Meth. Appl. Sciences 3 (1993), no. 2, 145-170. MR 94e:65130

[11] Cercignani, C., Theory and application of the Boltzmann equation, Springer, (1988).

[12] P. Le Tallec and M. D. Tidriri, Convergence Analysis of Domain Decomposition Algorithms with Full Overlapping for the Advection-Diffusion Problems. Rapport de recherche INRIA no 2435, Octobre 1994 (57 pages).

[13] P. Le Tallec and M. D. Tidriri, Analysis of the explicit time marching algorithm. ICASE Report No. 96-45.

[14] L. Marini and A. Quarteroni, An iterative procedure for domain decomposition methods: a finite element approach. In [3] 129-143. MR 90d:65196

[15] L. D. Marini and A. Quarteroni, A relaxation procedure for domain decomposition methods using Finite Elements, Numer. Math. 55, (1989) 575-598. MR 90g:65150

[16] A. Quarteroni, G. Sacchi Landriani and A Valli, Coupling of Viscous and Inviscid Stokes Equations via a Domain Decomposition Method for Finite Elements, Technical report UTM89-287 (Dipartimento di Mathematica, Universita degli Studi di Trento, 1989).

[17] Ph. Rostand, B. Stoufflet, Finite volume Galerkin methods for viscous gas dynamics, Rapport de recherche INRIA no 863, Juillet 1988.

[18] M. D. Tidriri, Couplage d'approximations et de modèles de types différents dans le calcul d'écoulements externes, PhD thesis, University of Paris IX, 1992. MR 96d:65202

[19] M. D. Tidriri, Domain Decomposition for Incompatible Nonlinear Models. INRIA Research Report RR-2378, October 1994.

[20] M. D. Tidriri, Domain decomposition for compressible Navier-Stokes equations with different discretizations and formulations. J. Comp. Phy. 119, 271-282 (1995). CMP 98:09

[21] Y. A. Kuznetsov, Overlapping Domain Decomposition Methods for Parabolic Problems. In [8]. CMP 94:08 
[22] H. Blum, S. Lisky and R. Rannacher, A domain decomposition algorithm for parabolic problems, Preprint 02-08, Interdisziplinaeres Zentrum fuer Wissenschaftliches Rechen, Universitaet Heidelberg, 1992; A domain splitting algorithm for parabolic problems, Computing 49 (1992), 11-23; MR 93f:65071

InRIA, Domaine de Voluceau Rocquencourt, B.P. 105, Le Chesnay Cedex, France

E-mail address: Partrick.LeTallec@inria.fr

Iowa State University, Department of Mathematics, 400 Carver Hall, Ames, IA 50011

E-mail address: tidriri@iastate.edu 